\title{
THE DETECTION OF VARIATION IN HOST SUSCEPTIBILITY IN DILUTION COUNTING EXPERIMENTS
}

\author{
By P. ARMITAGE \\ Statistical Research Unit of the Medical Research Council, \\ London School of Hygiene and Tropical Medicine \\ AND C. C. SPICER \\ Central Public Health Laboratory, Colindale
}

(With 2 Figures in the Text)

\section{INTRODUCTION}

This paper is concerned with the statistical analysis of experiments in which a series of doses containing different numbers of particles (typically micro-organisms) are administered to groups of host organisms, and the proportion of host organisms infected at each dilution is recorded. Isaacs (1956) has reviewed the general problem of counting virus particles, including the use of the dilution method.

It is assumed that, for each host, a proportion $p$ of the particles will initiate a detectable infection and that the particles act independently. The proportion $p$, which may be regarded as the probability that a particle chosen at random will be infective, may vary from host to host. We shall refer to $p$ as the susceptibility of the host. This model has been proposed by Bald (1937) for the infection of plants by viruses, by Moran $(1954 a, b)$ for the titration of viruses in eggs, and by Druett (1952) and Peto (1953) for the infection of animals by bacteria. If $p$ is the same for each host, the expected proportion of hosts remaining uninfected when inoculated at a concentration of $\lambda$ particles per dose is

$$
P=e^{-\lambda p} \text {. }
$$

We shall refer to this as the exponential model. If the values of $\lambda$ are known (as they are, approximately, in bacterial invasion), and those of $P$ are estimated by the observed proportions of uninfected hosts, the dose-response curve (1) can be fitted, and $p$ estimated, by essentially the same methods as are used in the dilution method of estimating bacterial counts (Finney, 1952, §21.5). One of many alternative methods of obtaining the maximum-likelihood solution is given by Peto.

If, as in most virus titrations, the values of $\lambda$ are unknown but their ratios are known, the most we can do is to estimate $\lambda_{0 p}$, where $\lambda_{0}$ is the number of particles at a particular dilution-say, in the undiluted inoculum.

If $p$ varies from host to host, with a distribution whose density function is $f(p)(0 \leqslant p \leqslant 1)$, the probability that a host is uninfected is

$$
P=\int_{0}^{1} e^{-\lambda p} f(p) d p
$$


The curves formed by $P$ as a function of $\lambda$, or $\log \lambda$, are flatter, broadly speaking, than those given by (1). Moran (1954a) has developed this model, assuming a Type III distribution for $p$, and shown how to obtain the maximum-likelihood solution for the unknown parameters. (The Type III distribution is defined over the range $0 \leqslant p \leqslant \infty$, which is unrealistic since $p$ cannot in fact exceed unity, but if the mean value of $p$ were very small the model might well be adequate.) Moran also proposes a rapid test for the tendency of the response curve to be flatter than the exponential curve (1), thus indicating host heterogeneity, i.e. variability in $p$. The two papers $(1954 a, b)$ provide tables enabling the test to be performed for titrations with dilution factors of $2,4, \sqrt{ } 10$ and 10 .

In the present paper we consider the nature of the departures of the response curve (2) from the exponential curve (1), and examine the extent to which the departures caused by particular functions $f(p)$ will be detected by Moran's test. We also compare the efficiency of Moran's test with that of an alternative test for heterogeneity.

\section{DEPARTURES FROM EXPONENTIALITY}

It is convenient to write the expression (2) in the form

$$
P=\int_{-\bar{p}}^{1-\bar{p}} e^{-\lambda(\bar{p}+h)} f(\bar{p}+h) d h,
$$

where $\bar{p}=$ the mean value of $p$ in the distribution and $h=p-\bar{p}$. Expanding the function inside the integral we have

$$
\begin{aligned}
P & =\int_{-\bar{p}}^{1-\bar{p}} e^{-\lambda \bar{p}}\left\{1-\lambda h+\frac{\lambda h^{2}}{2 !}-\ldots\right\} f(\bar{p}+h) d h \\
& =e^{-\lambda \bar{p}}\left\{1+\frac{\lambda^{2} \mu_{2}}{2 !}-\frac{\lambda^{3} \mu_{3}}{3 !}+\ldots\right\},
\end{aligned}
$$

where $\mu_{k}$ is the $k$ th moment of $f(p)$ about the mean. It is now obvious that as the average number of particles in the inoculum to be tested becomes small $(\lambda \rightarrow 0)$, or as the variation in susceptibility declines, the probability of a sterile culture becomes more and more closely described by the exponential law.

On the other hand, when the expected number of particles, $\lambda$, or the variation in susceptibility becomes large, the discrepancy between the true law and the exponential increases. The general character of the discrepancy is shown in Figs. 1 and 2 in which (writing $\ln$ for $\log$ to base $e) \ln (-\ln P$ ) is plotted against $\lambda$ for some non-exponential curves (the scale for $\lambda$ being logarithmic).

From a practical point of view it is essential that the departure from the exponential form should occur in regions where it has a reasonable probability of being detected. For instance, if discrepancies occur only at dilutions where the probability of a sterile inoculum is about $1 \%$ or less, they are not likely to be picked up unless large numbers of experimental units are available.

Returning to the expression (3) giving $P$ in terms of the moments of the distribution of susceptibility, it can be seen that the optimum conditions for testing the adequacy of the exponential law depend on the relative values of $\bar{p}, \lambda$ and the 
moments $\mu_{k}$. The latter are bound to be fairly small since $p$ is always less than unity, and furthermore, for most distributions over the range $(0,1)$ which are likely to be encountered in practice, the higher order moments tend to decrease rapidly so that, as an approximation valid in many cases we can write

$$
P \doteqdot e^{-\lambda p}\left(1+\frac{1}{2} \lambda^{2} \mu_{2}\right)
$$

If $\bar{p}$ is near to 1 , then in the region of $P=0 \cdot 5, \lambda$ is approximately $0 \cdot 7$, and as the value of $\mu_{2}$ can never be more than 0.25 and will usually be much less, the correction term involving $\mu_{2}$ cannot alter the value of $P$ by more than about $10 \%$.

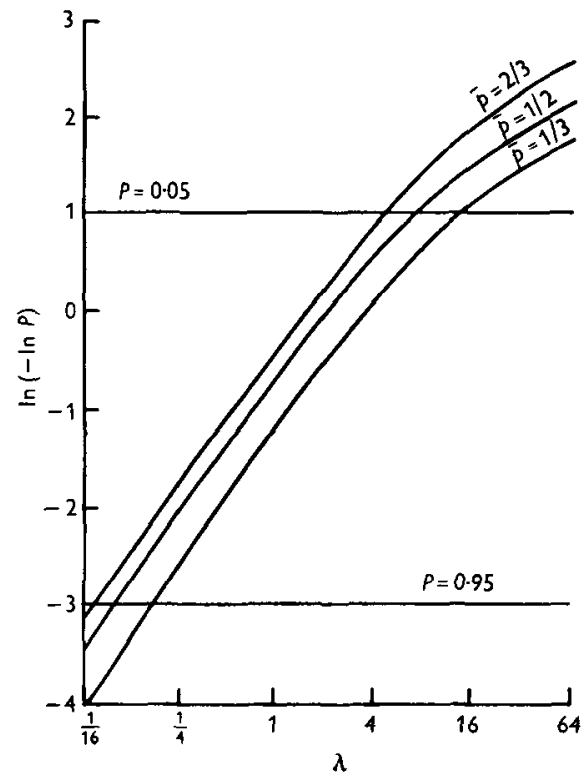

Fig. 1

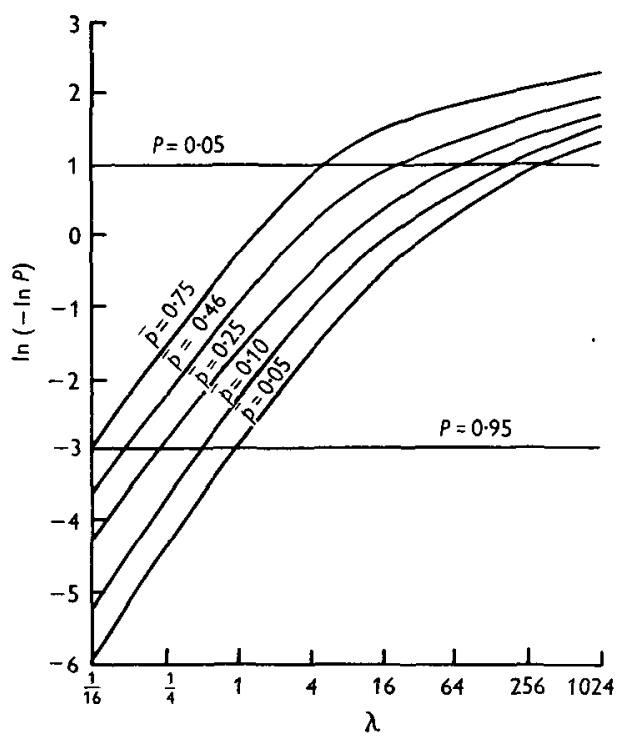

Fig. 2

Fig. 1. Curves showing relation between $\ln (-\ln P$ ) and (on a logarithmic scale) $\lambda$, when host susceptibility follows $B$-function distributions with means $2 / 3,1 / 2,1 / 3$. If there were no host variability the relation would be linear.

Fig. 2. Relation between $\ln (-\ln P$ ) and (on a logarithmic scale) $\lambda$, when host susceptibility follows truncated exponential distributions with various means.

As an illustration consider the case of a $B$-distribution having a mean of $0 \cdot 25$ and a mode at 0 , its frequency function being

$$
f(p)=3(1-p)^{2} .
$$

The first two moments about the mean are

so that

$$
\begin{aligned}
& \mu_{2}=0.0375, \\
& \mu_{3}=0.00625,
\end{aligned}
$$

When $\lambda=3$ about 250 observations would be required before the divergence from the exponential value had an even chance of being detected, and when $\lambda=1$ the difference is so small that it would not be detected in any practicable experiment. 
If the hypothesis of variation in susceptibility of the hosts is correct, and if the higher moments of the distribution are negligible, then it might be worth using the approximation to $P$ given by (4) instead of Moran's negative binomial model. The expression for $P$ may be written

$$
P=e^{-\alpha \gamma_{0}}\left(1+\frac{1}{2} \alpha^{2} \gamma_{0}^{2} \mathrm{~V}\right),
$$

where $\alpha$ is the dilution, $\gamma_{0}$ is the value of $\lambda \bar{p}$ at the highest concentration, and $\sqrt{ } V$ is the ratio of the standard deviation of $p$ to its mean.

The usual maximum-likelihood procedure will yield estimates of $\gamma_{0}$ and $V$, but the calculations are too clumsy to be of much practical use. It is perhaps worth noting that this approach throws some light on the information which each dilution yields about the value of $\mu_{2}$, on the assumption that $\lambda$ and $\bar{p}$ are known. Differentiating the likelihood function in the usual way it is found that the information about $\mu_{2}$ at a single level is

$$
I_{\mu_{2}}=\frac{n \lambda^{4} e^{-\lambda \bar{p}}}{4\left(1+\frac{1}{2} \lambda^{2} \mu_{2}\right)\left\{1-e^{-\lambda \bar{p}}\left(1+\frac{1}{2} \lambda^{2} \mu_{2}\right)\right\}}
$$

The information is the reciprocal of the sampling variance of the maximumlikelihood estimate of $\mu_{2}$. If $\mu_{2}$ is very small this function has a maximum (and hence the sampling variance has a minimum) at the point where the inoculum contains about four infective particles, so that the optimum dilution for detecting small departures from the exponential form is that which gives about $98 \%$ positive inocula.

A number of different kinds of distribution of susceptibility were investigated in detail to find out the effect they had in causing deviations from the exponential law. The two general types of distribution used were, first, $B$-functions which give bell-shaped distributions in the range $(0,1)$, and, secondly, truncated exponential curves. The latter bear some resemblance to the $\Gamma$-function considered by Moran but are more realistic as they provide a group of highly skewed distributions over the range $(0,1)$, which can be integrated to give an explicit expression for the probability of a negative inoculum. On the other hand, the $B$-functions cannot be conveniently integrated and the required probabilities are more easily obtained by numerical methods. ${ }^{*}$ Examples of the results of this investigation are given in Fig. 1 for the $B$-functions, and in Fig. 2 for the truncated exponentials.

The three $B$-functions used to obtain the results shown in Fig. 1 had the general frequency function

$$
f(p)=\frac{p^{l-1}(1-p)^{m-1}}{B(l, m)},
$$

the values of $l$ and $m$ being varied to give: (1) a positively skew, (2) a symmetrical, and (3) a negatively skew distribution. The values of $l$ and $m$ used, together with

* The exact values can be obtained rather tediously by integration by parts. An alternative method would have been to express $P$ in terms of the confluent hypergeometric function (cf. Irwin, 1930); the required values of $P$ for certain values of $\lambda$ can be obtained almost immediately from published tables of this function, and for other values of $\lambda$ by simple extension of these tables. The exact values of $P$ agree well with those obtained by numerical integration. 
the characteristics of the distributions, are given in Table 1. Numerical integration of the $B$-functions to obtain the probability of negative inocula did not demand great accuracy, and the method adopted as most simple and satisfactory was the repeated Simpson formula (Whittaker \& Robinson, 1944).

Table 1. Constants of B-functions used to represent distributions of susceptibility

$\begin{array}{rrrrc} & l & m & \text { Mean } & \text { Variance } \\ 1 & 2 & 4 & 1 / 3 & 2 / 63 \\ 2 & 3 & 3 & 1 / 2 & 1 / 28 \\ 3 & 4 & 2 & 2 / 3 & 2 / 63\end{array}$

Table 2. Formulae of truncated exponentials used to represent distributions of susceptibility, together with some of their characteristics

$\begin{array}{lccc} & \begin{array}{c}\text { Negatively } \\ \text { skew } \\ f(p) d p\end{array} & \begin{array}{c}\text { Positively } \\ \text { skew } \\ k e^{-k(1-p)}\end{array} & \text { Rectangular } \\ 1-e^{-k} & \frac{k e^{-k p}}{1-e^{-k}} d p & d p \\ P=\int_{0}^{1} f(p) e^{-\lambda p} d p & \frac{k}{k-\lambda} \frac{e^{(k-\lambda)}-1}{e^{k}-1} & \frac{k}{k+\lambda} \frac{1-e^{-(k+\lambda)}}{1-e^{-k}} & \frac{1}{\lambda}\left(1-e^{-\lambda}\right) \\ \text { Mean } & \frac{1}{1-e^{-k}}-\frac{1}{k} & \frac{1}{k}-\frac{1}{e^{k}-1} & \frac{1}{2} \\ \text { Variance } & \frac{1}{k^{2}}-\frac{e^{k}}{\left(e^{k}-1\right)^{2}} & \frac{1}{k^{2}}-\frac{e^{k}}{\left(e^{k}-1\right)^{2}} & \frac{1}{12}\end{array}$

It can be seen at once from Fig. 1 that variations in susceptibility of this kind have little effect on the exponential curve and are not likely to be detected in practice.

The truncated exponential curves used were of two types, one with a mode at unity and negatively skew, the other with a mode at zero and positively skew. Their general formulae and characteristics are given in Table 2. The rectangular distribution occurs as a degenerate case of these truncated exponentials. In Fig. 2 the relationship between $\ln (-\ln P)$ and $\lambda$ is given for truncated exponential distributions of susceptibility with various mean values of $p$. The departures from the exponential form are more marked than was the case for the bell-shaped $B$-distributions, but even here the main departures from exponentiality occur at very high rates of infection where they are difficult to detect experimentally. On the whole, discrepancies will not be noticed unless the average susceptibility is low, $\bar{p}$ having a value of $0 \cdot 2-0 \cdot 1$ or less.

The test for detection of host variability given by Moran (1954a) is based on the quantity

$$
T=\Sigma r_{i}\left(n-r_{i}\right),
$$

where $n$ experimental units are used at each dilution and $r_{i}$ is the number infected at the $i$ th dilution. The results of using Moran's test for four-fold series are summarized in Table 3. The data relate only to truncated exponentials, as the test 
would be even less sensitive when applied to the $B$-function data. The results were calculated by assuming that $T$ was normally distributed with variance given by Moran's formula. It is then possible, using tables of the normal curve, to estimate how often $T$ will differ significantly from the value given by the exponential law. The calculations for a four-fold series when the distribution of $p$ is rectangular may be taken as an example. If the number of replicates at each dilution is 10, Moran's (1954b) Table 1 gives the expected value of $T$ as 45 , with standard deviation $12 \cdot 04$, so that in this case values of $T$ greater than $45+(1 \cdot 65)(12.04)=64.9$ will be judged significant by a one-sided test at the $5 \%$ level. Now for a rectangular distribution of susceptibility the expected value of $T$ is 57 , and its standard error is 14 , so, assuming $T$ to be normally distributed, the probability that it will exceed 64.9 is that corresponding to the normal deviate $(65-57) / 14=0.56$. From a table of the normal distribution this probability is found to be $0 \cdot 29$. If the number of replicated inocula had been 40 , the probability would have been 0.63 . In general it can be

Table 3. Table showing the probability that dilution series with 10 and 40 inocula at each level will show significant departures from exponentiality as judged by Moran's $T$-test (using the one-sided $5 \%$ significance level). The underlying host susceptibility follows a truncated exponential distribution with the means shown

$\begin{array}{ccc}\text { Mean } & n=10 & n=40 \\ 0.05 & 0.48 & 0.92 \\ 0 \cdot 10 & 0 \cdot 49 & 0.93 \\ 0 \cdot 25 & 0.48 & 0.92 \\ 0.46 & 0.33 & 0 \cdot 72 \\ 0.50 & 0.29 & 0 \cdot 63 \\ 0.75 & 0 \cdot 17 & 0.36\end{array}$

seen from Table 3 that the test is not at all sensitive to statistical variations in susceptibility of the types considered here unless the average susceptibility is low.

\section{AN ALTERNATIVE APPROACH}

If $X=\lambda p$ is the mean number of infective particles per dose given to a particular host whose susceptibility is $p$, the probability of infection for that host is, from (1),

$$
F_{1}(X)=1-e^{-X} .
$$

This is a monotonic function, increasing from 0 to 1 as $X$ ranges from 0 to $\infty$, and may therefore be regarded as the cumulative distribution function of a random variate, which will be denoted by $x$.

Suppose now that $p$ varies from host to host, according to the density function $f(p)$. Define

$$
F_{2}(p)=\int_{0}^{p} f(t) d t
$$

as the cumulative distribution of the random variate $p$. 
By (2), the probability of infection, when $p$ varies in this way, is

$$
\begin{aligned}
F_{3}(\lambda) & =1-\int_{0}^{1} e^{-\lambda t} f(t) d t \\
& =\int_{0}^{1}\left(1-e^{-\lambda t}\right) f(t) d t,
\end{aligned}
$$

which is the formula for the distribution function of a random variate $z=x / p$, where $x$ and $p$ are independent random variates from the distributions $F_{1}$ and $F_{2}$, respectively. It will be convenient to refer to the frequency distribution of $z$ as the tolerance distribution, as is customary for other forms of quantal response data.

Since $z=x / p$, where $x$ and $p$ are independent,

$$
\log z=\log x-\log p,
$$

where $\log x$ and $\log p$ are independent. Hence

$$
\sigma_{\log z}^{2}=\sigma_{\log x}^{2}+\sigma_{\log p}^{2}
$$

where $\sigma_{\log z}^{2}$ is the variance of $\log z$, and so on. Since the variance of $\log z$ is related to the steepness of the cumulative distribution (7) (the curve becoming steeper as the variance decreases), (8) is a precise formulation of the way in which the response curve flattens as $\sigma_{\log p}^{2}$ increases.

The distribution of $\log x$ has been studied by Irwin (1942). From Irwin's equation (13), we find (taking logarithms to base 10)

$$
\sigma_{\log x}^{2}=\left(\log _{10} e\right)^{2}\left(\pi^{2} / 6\right)=0 \cdot 31025 \text {. }
$$

Hence, from (8),

$$
\sigma_{\log z}=\sqrt{ }\left\{\sigma_{\log p}^{2}+0 \cdot 31025\right\} \text {. }
$$

The exponential response curve (1) is appropriate if $p$, and therefore $\log p$, is the same for all hosts, i.e. if $\sigma_{\log p}^{2}=0$. Under these circumstances $\sigma_{\log z}=\sqrt{ } 0 \cdot 31025=$ 0.5570 . The problem of detecting heterogeneity in $p$ is therefore, from this point of view, the problem of determining whether or not $\sigma_{\log z}$ is greater than 0.5570 , and one might expect it to be amenable to treatment by standard methods of analysis of quantal response data.

\section{METHODS OF ESTIMATING $\sigma$}

Many methods have been proposed for the estimation of the mean or standard deviation of a tolerance distribution from a series of quantal responses at different doses (cf. Finney, 1952, chapter 20). Some of these methods assume a particular type of distribution, such as the normal or logistic. As Irwin (1942) has shown, the cumulative distribution of $\log x$, when the exponential model holds, is fairly similar to a normal distribution, and one might expect the random variate $\log z=\log x-\log p$ also to be approximately normally distributed.

One approach, then, might be to regard $\log z$ as being approximately normally distributed, and to use probit analysis, assuming a linear relationship between the probit of $1-P$ and $\log \lambda$. The standard deviation $\sigma_{\log z}$ would be estimated by the reciprocal of the slope of the probit line. The expected slope on the exponential model would depend on the choice of the values of $\lambda$. Peto (1953) has shown that the expected slope, as measured by the tangent of the curve when $P=0 \cdot 5$, is $2 \cdot 00$, 
which is fairly near the reciprocal of 0.5570 , the value of $\sigma_{\log z}$ on this model $(1 / 0 \cdot 5570=1 \cdot 80)$.

Another approach to the estimation of $\sigma_{\log z}$ would be to assume a particular functional form for $f(p)$ and to estimate the parameters of the distribution by an efficient method such as maximum likelihood. Moran (1954a) has given the details of one such model. However, if the distribution of $p$ is concentrated at one value, as in the exponential model, the value of Moran's $l$ (the parameter determining $\left.\sigma_{\log z}\right)$ is infinite, and it seems likely that the iterative procedure leading to the maximum-likelihood solution would frequently not converge.

There is probably a place for a rapid test based on some short-cut method of estimating $\sigma_{\log z}$, and Moran's test may be regarded as an example of this approach. For two-fold dilution series, Moran shows that the expected value of $T$, on the exponential model, is $n(n-1)$. (For other dilution factors, see Moran, 1954b). Now, $T$ provides an estimate of $\sigma_{\log z}$, and its properties as an estimator, for small departures from the exponential model, will be examined in $\S 5$. If the response curve of infection rate against $\log \lambda$ had been a cumulative normal distribution, so that $\log z$ were a normal deviate, the expected value of $T$ would have been

$$
E(T)=\frac{n(n-1)}{\log _{10} 2} \int_{-\infty}^{\infty} P(1-P) d \log z=\frac{n(n-1)}{\log _{10} 2} \frac{\sigma_{\log z}}{\sqrt{ } \pi}=\frac{n(n-1) \sigma_{\log z}}{0.5336}
$$

(Van der Waerden, 1940, equation (6)). On the exponential model,

and thus from (11),

$$
\begin{gathered}
\sigma_{\log z}=\sigma_{\log x}=0.5570, \\
E(T)=1.044 n(n-1),
\end{gathered}
$$

which agrees well with Moran's exact expression, $n(n-1)$.

Moran's statistic $T$ does not seem to have been widely discussed as a general method of estimating the standard deviation of a tolerance distribution, and it is worth considering whether any other easily calculable statistics have been proposed, which may be more efficient as estimators of $\sigma_{\log z}$, and therefore more powerful in detecting heterogeneity in $p$, than is $T$. One alternative statistic is considered in $\$ 5$.

\section{THE SPEARMAN-KÄRBER METHOD}

This method (for reference to which, see Finney, 1952, §20.6) has often been advocated for the estimation of the mean of a tolerance distribution, and has been adapted by Epstein \& Churchman (1944) for the estimation of higher moments, including the variance. Suppose that at the $i$ th dilution, where the number of infecting organisms is $\lambda_{i}$, there are $r_{i}$ infections out of $n_{i}$, and let $q_{i}=r_{i} / n_{i}$ and $\zeta_{i}=\log _{10} \lambda_{i}$; then an estimate of the cumulative probability between two adjacent doses is $\left(q_{i}-q_{i+1}\right)$. Hence, the mean of the tolerance distribution may be estimated by

$$
m=\Sigma\left(q_{i}-q_{i+1}\right)\left(\zeta_{i}+\zeta_{i+1}\right) / 2
$$

the summation being over a wide enough range of doses to cover the whole range of responses from 0 to $100 \%$. An estimate of the variance, $\sigma_{\log z}^{2}$, is given by

$$
s_{\log z}^{2}=\Sigma\left(q_{i}-q_{i+1}\right)\left(\frac{\zeta_{i}+\zeta_{i+1}}{2}-m\right)^{2}-c
$$


where $c$ is a Sheppard's correction term. If, as in most dilution series, the $\zeta_{i}$ are equally spaced, the logarithm of the dilution factor being $d$, the estimate of variance may be calculated as

$$
s_{\log z}^{2}=d^{2}\left(2 S_{2}-S_{1}-S_{1}^{2}-\frac{1}{12}\right),
$$

where $S_{1}=\sum_{i=0}^{k} q_{i}$ and $S_{2}=\sum_{j=0}^{k} \sum_{i=j}^{k} q_{i}$. In the summations the suffixes 0 and $k$ refer to the highest and lowest 'doses' (i.e. lowest and highest dilutions) respectively. It is assumed in the derivation of (12) that all dilutions beyond the range used would have given $q=0$ at one extreme and $q=1$ at the other. A correction for bias in $s_{\log z}^{2}$ is given by Cornfield \& Mantel (1950), but will usually be too small to be worth making. The variance of $s_{\log z}^{2}$ is*

$$
\operatorname{var}\left(s_{\log z}^{2}\right)=4 d^{2} \Sigma\left(\zeta_{i}-\mu\right)^{2} P_{i} Q_{i} / n_{i},
$$

where $Q_{i}$ is the expected infection rate at the $i$ th dilution, $P_{i}=1-Q_{i}$, and $\mu$ is the true mean of the tolerance distribution. Now, on the exponential model, the expression on the right-hand side of (13) varies slightly according to the position of $\mu$ relative to the serial dilutions; but, if the $n_{i}$ are all equal to $n$, it is always close to the corresponding integral, which can be shown to be equal to

$$
0 \cdot 40996 d / n \text {. }
$$

As a test of the null hypothesis that there is no variability in $p$, then, we could calculate $\frac{n\left(s_{\log z}^{2}-0 \cdot 31025\right)^{2}}{0 \cdot 40996 d}$ and regard this as a $\chi^{2}$ variate on 1 degree of freedom.

Since the test based on $\chi$ is one-sided (being designed to detect values of $s_{\log z}^{2}$ larger, but not smaller, than its expected value), the tabulated probabilities of $\chi^{2}$

\begin{tabular}{|c|c|c|c|c|c|}
\hline \multirow[b]{2}{*}{ Dilution } & \multirow[b]{2}{*}{$i$} & \multicolumn{3}{|c|}{ Proportions } & \multirow[b]{2}{*}{$\begin{array}{c}\text { Cumulative } \\
\text { sum of } \\
q_{i}\end{array}$} \\
\hline & & $\begin{array}{l}\text { No. uninfected } \\
\text { out of } 40 \\
40-r_{i}\end{array}$ & $\begin{array}{l}\text { Uninfected } \\
1-\left(r_{i} / 40\right)\end{array}$ & $\begin{array}{c}\text { Infected } \\
q_{i}=r_{i} / 40\end{array}$ & \\
\hline 1 & 0 & 0 & 0 & $1 \cdot 000$ & $4 \cdot 450$ \\
\hline $2^{-1}$ & 1 & 5 & $0 \cdot 125$ & 0.875 & $3 \cdot 450$ \\
\hline $2^{-2}$ & 2 & 8 & $0 \cdot 200$ & 0.800 & $2 \cdot 575$ \\
\hline $2^{-3}$ & 3 & 15 & 0.375 & $0 \cdot 625$ & $1 \cdot 775$ \\
\hline $2^{-4}$ & 4 & 21 & 0.525 & 0.475 & $1 \cdot 150$ \\
\hline $2^{-5}$ & 5 & 30 & 0.750 & $0 \cdot 250$ & 0.675 \\
\hline $2^{-6}$ & 6 & 32 & 0.800 & $0 \cdot 200$ & 0.425 \\
\hline $2^{-7}$ & 7 & 35 & 0.875 & 0.125 & 0.225 \\
\hline $2^{-8}$ & 8 & 36 & 0.900 & $0 \cdot 100$ & $0 \cdot 100$ \\
\hline & & & & $4 \cdot 450$ & $14 \cdot 825$ \\
\hline
\end{tabular}
should be halved.

Table 4. Data of Parker, quoted by Moran (1954a)

As an example, the data of Parker, quoted by Moran (1954a), are shown in Table 4. Following Moran, we adjust the numbers of eggs at the first two dilutions from 38 and 39 , to 40 , so as to make the $n_{i}$ equal.

* Cornfield \& Mantel erroneously have a factor 2 instead of 4. 
From (12), putting $d=\log _{10} 2=0 \cdot 30103$,

$$
\begin{aligned}
s_{\log z}^{2} & =(0.30103)^{2}(29 \cdot 6500-4 \cdot 4500-19 \cdot 8025-0.0833) \\
& =0 \cdot 48157 ; \\
\operatorname{var}\left(s_{\log z}^{2}\right) & =(0 \cdot 40996)(0 \cdot 30103) / 40 \\
& =0.003086 \\
\chi^{2} & =(0.48157-0.31025)^{2} /(0.003086)=9.5 \quad(P=0.001) .
\end{aligned}
$$

Application of Moran's test gives:

$$
\begin{gathered}
T=2080, \quad E(T)=1560, \text { s.æ. }(T)=141 \cdot 6 \\
\{T-E(T)\} / \text { s.E. }(T)=3 \cdot 675 \\
\chi^{2}=(3 \cdot 675)^{2}=13 \cdot 5 \quad(P=0 \cdot 0001) .
\end{gathered}
$$

The Spearman-Kärber method provides an estimate of $\sigma_{\log z}^{2}$ and therefore (from (10)) of $\sigma_{\log p}^{2}$. The estimate of $\sigma_{\log p}^{2}$ will be

$$
s_{\log p}^{2}=s_{\log z}^{2}-0 \cdot 31025,
$$

which, for the example above, gives

$$
s_{\log p}^{2}=0 \cdot 1713,
$$

with a standard error of $\sqrt{ } 0 \cdot 003086=0 \cdot 0556$.

To estimate $\sigma_{\log p}^{2}$ from $T$, we may assume that the response curve, of $P$ against $\log \lambda$, approximates to a cumulative normal distribution. From (11), an estimate of $\sigma_{\log z}^{2}$ is

$$
s_{\log z}^{\prime 2}=(0 \cdot 5336)^{2}\left\{\frac{T}{E(T)}\right\}^{2}=(0 \cdot 2847)\left\{\frac{T}{E(T)}\right\}^{2},
$$

which in this example gives

Approximately,

$$
s_{\log z}^{\prime 2}=(0 \cdot 2847)(1 \cdot 7778)=0 \cdot 5061 .
$$

$$
\operatorname{var}\left(s_{\log z}^{\prime 2}\right)=\frac{4(0 \cdot 2847)^{2}}{\{E(T)\}^{2}} \operatorname{var}(T)=(0 \cdot 3242)\left\{\frac{\text { s.E. }(T)}{E(T)}\right\}^{2}
$$

which in this example gives

$$
\operatorname{var}\left(s_{\log z}^{\prime 2}\right)=(0 \cdot 3242)(0 \cdot 09071)^{2}=0 \cdot 002668 .
$$

The estimate of $\sigma_{\log p}^{2}$ is, therefore,

$$
s_{\log p}^{\prime 2}=s_{\log z}^{\prime 2}-0 \cdot 31025=0 \cdot 1959,
$$

with a standard error of $\sqrt{ } 0 \cdot 002668=0 \cdot 0517$. The two methods agree well.

\section{RELATIVE EFFICIENCIES OF THE TWO TESTS}

We now investigate the relative efficiencies of the two tests in detecting heterogeneity in $p$; a more precise indication of what we mean by 'relative efficiency' is given a little later. Suppose the mean value of $p$ is $\bar{p}$. Under the null hypothesis, the expected proportion of uninfected hosts, at a dose of $\lambda$ particles, is (from (1))

where $\gamma=\lambda \bar{p}$.

$$
P=e^{-\gamma},
$$


As an alternative hypothesis, Moran assumes that $p$ follows a Type III distribution, with density function

$$
f(p)=e^{-p / \bar{p} V}(p / \bar{p} V)^{(1 / V)-1} /(\bar{p} V) \Gamma(1 / V),
$$

where* Moran's $l=1 / V$ and his $a=\bar{p} V$. The mean of the distribution is $\bar{p}$ and the ratio of the standard deviation to the mean is $\sqrt{ } V$. From (2), the expected proportion of uninfected hosts is now

$$
P=(1+\gamma V)^{-(1 / V)},
$$

which, as would be expected, tends to (15) as $V \rightarrow 0$.

We shall consider the effect on the expected values of $T$ and $s_{\log z}^{2}$, for small departures from the null hypothesis (i.e. for small positive values of $V$ ), and for large $n$. From (17),

and

$$
\ln P=-(1 / V) \ln (1+\gamma V),
$$

$$
\frac{\partial \ln P}{\partial V}=-\frac{\gamma}{V(1+\gamma V)}+\frac{\ln (1+\gamma V)}{V^{2}} .
$$

Expanding in powers of $V$, we find $\dagger$

$$
\frac{\partial \ln P}{\partial V}=\frac{\gamma^{2}}{2}+O(V)
$$

and hence

$$
\frac{\partial(P Q)}{\partial V} \sim \frac{\gamma^{2} P(1-2 P)}{2} \text {. }
$$

Now,

$$
E(T)=n(n-1) \Sigma P_{i} Q_{i} \sim n^{2} \Sigma P_{i} Q_{i} \quad \text { for large } n,
$$

whence

$$
\frac{\partial}{\partial V}\{E(T)\}_{V=0} \sim \frac{n^{2} \Sigma \gamma_{i}^{2} P_{i}\left(1-2 P_{i}\right)}{2}=\frac{n^{2} \Sigma \gamma_{i}^{2} e^{-\gamma_{i}}\left(1-2 e^{-\gamma_{i}}\right)}{2} .
$$

The result of the summation, for any particular dilution factor, will (as in (13)) vary slightly according to the set of values of $\gamma_{i}$ used; but it will always be close to the corresponding integral

$$
\frac{\log _{10} e}{d} \int_{-\infty}^{\infty} e^{2 t} e^{-e^{t}}\left(1-2 e^{-e^{t}}\right) d t=\frac{\log _{10} e}{2 d}=\frac{0 \cdot 21715}{d},
$$

$=0.7213$ for 2 -fold dilutions, since $\alpha=0.30103$.

On the null hypothesis, the variance of $T$ is (cf. Moran, 1954b)

$$
\begin{aligned}
\operatorname{var}(T) & =n(n-1)\left\{(n-1) \Sigma P_{i} Q_{i}-(4 n-6) \Sigma P_{i}^{2} Q_{i}^{2}\right\} \\
& \sim n^{3}\left(\Sigma P_{i} Q_{i}-4 \Sigma P_{i}^{2} Q_{i}^{2}\right) \quad \text { for large } n,
\end{aligned}
$$

which, as Moran shows, for two-fold dilutions approximates to

$$
n^{3}\{1-4(0 \cdot 169925)\}=0 \cdot 3203 n^{3} .
$$

* There seems to be a discrepancy between equations (5) and (6) of Moran (1954a). We have assumed that his (5) should be written

$$
f(p)=e^{-p / a}(p / a)^{l-1} / a \Gamma(l) .
$$

$\dagger$ This expression is readily obtained from the approximation (4), in which no particular functional form for $f(p)$ is assumed, but higher moments than the second are neglected. 
Hence,

$$
\begin{aligned}
R^{2}(T) & =\left[\frac{\partial}{\partial V}\{E(T)\}\right]_{V=0}^{2} / \operatorname{var}(T) \\
& \sim\left(0.3607 n^{2}\right)^{2} / 0.3203 n^{3} \\
& =0.4062 n .
\end{aligned}
$$

$R(T)$ may be regarded as the rate of change, with respect to $V$, of the expectation of the normally distributed variate, $\chi$. Since $T$ is, for large $n$, asymptotically normally distributed, $R(T)$ is clearly related to the power of the significance test based on $T$ in detecting small changes in $V$. In fact, to compare the values of $n$ required, by two alternative tests, to provide the same power (i.e. probability of yielding a result significant at a given probability level) for small values of $V$, we need merely take the ratio of the two values of $R^{2}$. This ratio is called the asymptotic relative efficiency (A.R.E.) of one test in comparison with another. We proceed, therefore, to obtain the value of $R^{2}$ for the Spearman-Kärber test based on the statistic $s_{\log z}^{2}$.

We have, from (10),

\begin{tabular}{|c|c|}
\hline Now, for small $V$, & $\begin{aligned} \sigma_{\log p}^{2} & \sim\left(\log _{10} e\right)^{2} \sigma_{p}^{2} / \bar{p}^{2} \\
& =0 \cdot 1886 \mathrm{~V}\end{aligned}$ \\
\hline Hence & $E\left(s_{\log z}^{2}\right) \sim 0.31025+0.1886 \mathrm{~V}$, \\
\hline and & $\frac{\partial}{\partial V}\left\{E\left(s_{\log z}^{2}\right)\right\}_{V=0}=0 \cdot 1886$. \\
\hline
\end{tabular}

Now, for small $V$,

$$
E\left(s_{\log z}^{2}\right)=\sigma_{\log z}^{2}=0 \cdot 31025+\sigma_{\log p}^{2} .
$$

From (14), on the null hypothesis that $V=0$, and for two-fold dilutions,

Hence

$$
\begin{gathered}
\operatorname{var}\left(s_{\log z}^{2}\right)=(0.40996)(0.30103) / n \\
=0.12341 / n . \\
R^{2}\left(s_{\log z}^{2}\right)=\left[\frac{\partial}{\partial V}\left\{E\left(s_{\log z}^{2}\right)\right\}\right]_{V=0}^{2} / \operatorname{var}\left(s_{\log z}^{2}\right) \\
=(0.1886)^{2} n / 0.12341=0.2882 n .
\end{gathered}
$$

The A.R.E. of Moran's test in comparison with the Spearman-Kärber test is, therefore,

$$
\frac{R^{2}(T)}{R^{2}\left(s_{\log z}^{2}\right)}=\frac{0 \cdot 4062 n}{0 \cdot 2882 n}=1 \cdot 409 .
$$

The Spearman-Kärber test, therefore, requires samples about 1.4 times as large as are required by Moran's test, in order to achieve the same power in detecting very slight heterogeneity in $p$. The relative efficiency in detecting larger changes in $V$ is not necessarily the same as the A.R.E., but it would be surprising if Moran's test were appreciably less efficient than the Spearman-Kärber test for moderate values of $V$.

The A.R.E. has been calculated above for two-fold dilution series, but it can easily be seen to be independent of the dilution factor, if the slight fluctuations due to positioning of the dilutions are ignored. 
It appears to be impossible to investigate the efficiencies of these two tests in comparison with that based on the maximum-likelihood estimate, because of the difficulties already mentioned in connexion with this method (cf. §4). In fact, the expected values, on the null hypothesis, of the second derivatives of the likelihood function do not all converge. However, one would have expected the SpearmanKärber estimate to be fairly efficient, since it is known to be, under certain circumstances, fully efficient when the response curve of $1-P$ against $\log \lambda$ is logistic (Cornfield \& Mantel, 1950). As pointed out in $§ 4$, the exponential model leads to a curve similar to the cumulative normal, and this in turn is very similar to the logistic. One would therefore expect Moran's test to be fairly highly efficient.

\section{DISCUSSION}

We have shown in $\$ 2$ that quite appreciable variability in $p$ may exist and yet be unlikely to be detected by Moran's test. It is natural to suggest that Moran's test could be improved by giving more weight to observations at dilutions where most inocula are positive, as it is in this region that discrepancies occur. At higher dilutions the data are of exponential form to a very good degree of approximation and observations there are largely wasted. Two methods might be adopted: (i) to allot many more experimental units at low dilutions; (ii) to modify Moran's statistic $T$, for example, by calculating $\Sigma r_{i}\left(n-r_{i}\right)^{2}$ instead of $\Sigma r_{i}\left(n-r_{i}\right)$, where $r_{i}$ is the number of infected units and $n$ the number of inocula tested at each dilution. The disadvantage of the second suggestion seems to be that the value of $\Sigma r_{i}\left(n-r_{i}\right)^{2}$ will have a large variance which might vitiate the increased weight given to low values of $P$.

The other test considered in this paper, based on the Spearman-Kärber method, has been found to be even less sensitive than that of Moran. Two possible alternative approaches, which might be worth further investigation, are:

(1) a maximum-likelihood solution, involving some assumption about the functional form of $f(p)$, similar to that discussed by Moran (1954a);

(2) the use of probit analysis, on the assumption that the response curve may be approximated to by an integrated normal curve. The slope of the probit line would then be compared with its expected value, on the exponential model, of about 2 .

Some general observations on dilution counts arise from the foregoing discussion. First, it seems unlikely that any economical test of exponentiality will be possible if variation in susceptibility is small or if the average susceptibility is high. Secondly, it is possible that variations in susceptibility might be confused with variations in inoculum size if the method of administering it is at all inaccurate. The latter possibility is probably not important in the virological example discussed by Moran but has been encountered by one of the present writers in bacteriology. Finally, it appears that the general usefulness of dilution counting in virus studies is problematical unless the results are checked by more direct counting methods. So long as there is no sensitive method of testing departures from exponentiality there is always a possibility that appreciable host variability 
is present, and in that case we no longer estimate the virus 'count', but rather a composite figure depending on both the mean number of infective particles and the distribution of susceptibility among the hosts. For this reason, dilution counts of virus suspensions made at different times and places may not be in any way comparable.

\section{SUMMARY}

Variation in host susceptibility results in flattening of the quantal response curve obtained in dilution counting experiments. This departure from the exponential curve obtained with uniform hosts is found primarily at the lower dilutions, where the infection rates are high. The test proposed by Moran, for the detection of host variability, may easily fail to detect quite appreciable heterogeneity with the numbers of observations that are likely to be available in practice. Examination of the response curves corresponding to various theoretical distributions of susceptibility suggests that detection of heterogeneity is unlikely unless the probability that a particle can initiate infection is distributed with a low mean and considerable positive skewness.

The problem is related to that of estimating the standard deviation of a tolerance distribution from quantal response data. This suggests an alternative test, based on the Spearman-Kärber method, which, however, appears to be no better than Moran's test. Both methods provide estimates of the variability of the susceptibility distribution.

\section{REFERENCES}

BALD, J. G. (1937). The use of numbers of infections for comparing the concentration of plant virus suspensions. I. Dilution experiments with purified suspensions. Ann. appl. Biol. 24, 33-5.

Cornfietd, J. \& Mantel, N. (1950). Some new aspects of the application of maximum likelihood to the calculation of the dosage response curve. J. Amer. statist. Ass. 45, 181-210.

Druetr, H. A. (1952). Bacterial invasion. Nature, Lond., 170, 288.

Epstein, B. \& Chunchman, C. W. (1944). On the statistics of sensitivity data. Ann. math. Statist. 15, 90-6.

Finney, D. J. (1952). Statistical Method in Biological Assay. London: Griffin.

IRwIN, J. O. (1930). On the frequency distribution of the means of samples from populations of certain Pearson's types. Metron, 8 (4), 51-105.

IRwIN, J. O. (1942). The distribution of the logarithm of survival times when the true law is exponential. J. Hyg., Camb., 42, 328-33.

IsaAcs, A. (1956). Particle counts and infectivity titrations for animal viruses. Advanc. Virus. Res. 4 (in the Press).

Moran, P. A. P. (1954a). The dilution assay of viruses. I. J. Hyg., Camb., 52, 189-93.

Moran, P. A. P. (1954b). The dilution assay of viruses. II. J. Hyg., Camb., 52, 444-6.

Peto, S. (1953). A dose-response equation for the invasion of micro-organisms. Biometrics, 9, 320-35.

VAN DER WAERden, B. L. (1940). Biologisches Konzentrazionsauswirtung. Ber. sächs. Ges. (Akad.) Wiss. 92, 41-4.

Whittaker, E. T. \& Robinson, G. (1944). Calculus of Observations, 4th ed. London: Blackie. 